\title{
\begin{tabular}{l|l} 
Mitraries & DSpace@MIT
\end{tabular}
}

\author{
MIT Open Access Articles
}

\section{Miniaturized electrochemical sensor modified with aptamers for rapid norovirus detection}

The MIT Faculty has made this article openly available. Please share how this access benefits you. Your story matters.

Citation: Wang, Nan, Masaaki Kitajima, Kalaivani Mani, Elgar Kanhere, Andrew J. Whittle, Michael S. Triantafyllou, and Jianmin Miao. “Miniaturized Electrochemical Sensor Modified with Aptamers for Rapid Norovirus Detection." 2016 IEEE 11th Annual International Conference on Nano/Micro Engineered and Molecular Systems (NEMS) (April 2016).

As Published: http://dx.doi.org/10.1109/NEMS.2016.7758320

Publisher: Institute of Electrical and Electronics Engineers (IEEE)

Persistent URL: http://hdl.handle.net/1721.1/110673

Version: Author's final manuscript: final author's manuscript post peer review, without publisher's formatting or copy editing

Terms of use: Creative Commons Attribution-Noncommercial-Share Alike 


\title{
Miniaturized Electrochemical Sensor Modified with Aptamers for Rapid Norovirus Detection
}

\author{
Nan Wang ${ }^{1}$, Masaaki Kitajima ${ }^{2}$, Kalaivani Mani ${ }^{2}$, Elgar Kanhere ${ }^{1}$, Andrew J. Whittle ${ }^{3}$, \\ Michael S. Triantafyllou ${ }^{3}$, Jianmin Miao'(MJMMIAO@ntu.edu.sg) \\ ${ }^{1}$ Nanyang Technological University, 50 Nanyang Avenue, 639798, Singapore \\ ${ }^{2}$ Singapore-MIT Alliance for Research and Technology (SMART), 1 CREATE Way, 138602, Singapore \\ ${ }^{3}$ Massachusetts Institute of Technology, 77 Massachusetts Avenue, Cambridge, MA 02139, USA
}

\begin{abstract}
This paper presents a miniaturized MEMS (microelectromechanical systems)-based electrochemical aptasensor, which utilizes aptamer as the recognition element for simple, sensitive and rapid detection of norovirus. The novelty of this work is integration of micro fabrication technology with aptamers to develop a miniaturized and portable electrochemical sensor for environmental pathogen monitoring. The binding capability between aptamers and on-chip sensing electrodes was investigated and the performance of the proposed MEMS electrochemical aptasensor was evaluated with respect to sensor response to different titers of murine norovirus, a model of human norovirus. This research forms initial basis for the development of an electrochemical MEMS-aptasensor platform and its application for virus detection.
\end{abstract}

Keywords-Miniaturized aptasensor; MEMS electrochemical sensor; Norovirus determination

\section{INTRODUCTION}

Noroviruses (NoVs), a genetically diverse group of the Caliciviridae family, have caused numerous outbreaks of viral gastroenteritis worldwide as the infection can easily spread through person-to-person transmission and contaminated food or water. NoVs are very stable in the environment and highly contagious (i.e., require low infectious dose), warranting development of a real-time, on-site and rapid detection system. Nowadays, NoVs are generally diagnosed by the reverse transcription-polymerase chain reaction (RT-PCR) assays. However, conventional RT-PCR is rather complicated, which usually requires complex and time-consuming operations such as RNA extraction, RT reaction (cDNA synthesis) and PCR amplification [1], rendering it unsuitable for on-site and rapid detection of NoVs. Recently, development of electrochemical sensors with biological recognition elements, especially aptamers (DNA or RNA molecules) for microbial pathogen detection, has gained great attention [2]. Aptamers, capable of specifically binding to selected targets, have shown advantages over antibodies for biosensing applications. The unique threedimensional conformational change of aptamers upon targetanalyte binding enables the aptasensor to detect NoV particles with high specificity and sensitivity [3]. In the present study, we aimed to develop a miniaturized MEMS-based electrochemical aptasensor for rapid norovirus detection. We fabricated a MEMS electrochemical aptasensor and evaluated its performance with respect to sensor response to murine norovirus (MNV), an experimental model of human norovirus.

\section{MATERIALS AND METHODS}

\section{A. Virus and cells}

The prototype MNV strain, MNV-1 (clone CW1, ATCC PTA-5935) was propagated on RAW 264.7 (ATCC TIB-71) cell line monolayers with Dulbecco's modified Eagle medium (DMEM) containing 10\% fetal bovine serum. The propagated MNV particles were purified with gel chromatography (illustra MicroSpin S-300 HR Columns, GE Healthcare Life Sciences), and MNV titer was determined by plaque assay as previously described [4].

\section{B. Fabrication of sensor base}

Proposed MEMS electrochemical aptasensor consists of different layers as shown in Fig. 1, in which silicon ( $\mathrm{Si}, 500$ $\mu \mathrm{m})$ substrate serves as the sensor base. A layer of silicon dioxide $\left(\mathrm{SiO}_{2}, 1 \mu \mathrm{m}\right)$ deposited by plasma-enhanced chemical vapor deposition (PECVD) provided insulation for on-chip sensing electrodes. Before patterning with positive photoresist, the substrate was thoroughly washed by acetone solution and deionized (DI) water to remove the impurities. After that, five $\mu \mathrm{m}$ thickness of positive photoresist was spin-coated on the substrate and exposed under UV light (365 nm i-line). The substrate along with exposed photoresist was then immersed into sufficient amount of AZ400K photoresist developer. The developing was continued for $120 \mathrm{~s}$ with agitation. A layer of chromium (Cr, $50 \mathrm{~nm}$ ) was sputtered on the patterned photoresist by using magnetron sputtering system to promote the adhesion between silicon dioxide layer and upper electrode structure. Subsequently, a layer of gold (Au, $200 \mathrm{~nm}$ ) was attached on the Cr layer to function as base electrode layer, in which the middle semicircle and the outer semicircle are the working electrode and counter electrode respectively. Another layer of positive photoresist of $5 \mu \mathrm{m}$ thickness was then spincoated and patterned to define the location of reference electrode (the inner semicircle). A layer of silver together with silver chloride $(\mathrm{Ag} / \mathrm{AgCl}, 300 \mathrm{~nm})$ was sputtered to build a three-electrode configuration (Fig. 2) for electrochemical measurement. The contact pads of three electrodes were wired by using conductive epoxy and the entire sensor base was baked in the oven at $80{ }^{\circ} \mathrm{C}$ for three hours. Finally, nonconductive epoxy was used to cover the sensor base except for the section of three electrodes. Fig. 2 shows the photograph of proposed MEMS electrochemical aptasensor after fabrication. 


\section{Immobilization of aptamers on gold working electrode}

Aptamer immobilization on the gold working electrode through the affinity between gold and thiol was carried out according to a previous study [3], with slight modification. Prior to immobilization, the fabricated MEMS sensor base was thoroughly washed with DI water and then dried with nitrogen gas $\left(\mathrm{N}_{2}\right)$. The aptamer was immobilized on the electrode by drop casting $500 \mathrm{nM}$ HPLC-purified MNV-specific aptamer (AG3, aptamer nucleotide sequence described in Giamberardino et al. [3]) modified at the 5 ' position with a 6hydroxyhexyl disulfide group (HS-5'-AG3; Integrated DNA Technologies, USA) in $20 \mathrm{mM}$ Tris- $\mathrm{ClO}_{4}$ buffer ( $\mathrm{pH} \mathrm{8.6)}$ and incubating for 5 days at $4{ }^{\circ} \mathrm{C}$. Finally, the electrode was incubated with $1 \mathrm{mM} \beta$-mercaptoethanol (BME) in ethanol for 5 min to back-fill the empty spots of the electrode surface, thus reducing the non-specific adsorption onto the gold working electrode surface.

\section{Visualization of aptamer immobilization}

The thiolated AG3 aptamer modified at the 3' position with 6-carboxyfluorescein (FAM) (HS-5'-AG3-3'-FAM) was immobilized on the electrode following the procedure described above, and the electrode was rinsed with PBS to remove excess (i.e., non-immobilized) aptamers. The aptamerderived FAM fluorescence was observed under a Laser Scanning Fluorescent Confocal Microscope (Zeiss).

\section{E. Electrochemical measurements}

Electrochemical measurements, including cyclic voltammetry $(\mathrm{CV})$ and square wave voltammetry (SWV) were performed with a bench-top electrochemical workstation $(\mathrm{CH}$ Instruments 760E, TX, USA) linked to a computer (Fig. 3). Three electrodes of the MEMS aptasensor were separately connected to the electrochemical workstation through crocodile clips. Both $\mathrm{CV}$ and SWV experiments were performed in the solution of $25 \mathrm{mM}$ phosphate buffer $(\mathrm{pH}$ 7) containing $4 \mathrm{mM}$ $\mathrm{K}_{3}\left[\mathrm{Fe}(\mathrm{CN})_{6}\right]$ and $10 \mu \mathrm{M}\left[\mathrm{Ru}\left(\mathrm{NH}_{3}\right)_{6}\right] \mathrm{Cl}_{3}$ and all measurements were conducted at room temperature. The open-circuit potential of the system was measured prior to all electrochemical measurements to prevent sudden potentialrelated changes in the self-assembled monolayer. $\mathrm{CV}$ experiments were recorded with initial and final potential of $0.4 \mathrm{~V}$, switching potential of $-0.3 \mathrm{~V}$, and scan rate of $60 \mathrm{mV} / \mathrm{s}$. SWV experiments were recorded in the potential range of -0.3 to $0.4 \mathrm{~V}$ under the quiescent condition. Parameters of SWV experiment were chosen as step potential of $4 \mathrm{mV}$, amplitude of $5 \mathrm{mV}$ and frequency of $10 \mathrm{~Hz}$. The ferricyanide/ruthenium hexamine reporter system is able to enhance the electrochemical signal $[5,6]$, in which ruthenium hexamine $\left(\left[\mathrm{Ru}\left(\mathrm{NH}_{3}\right)_{6}\right]^{3+}\right)$, a cationic electron acceptor, undergoes redox reaction in the vicinity of working electrode. The ferricyanide $\left(\left[\mathrm{Fe}(\mathrm{CN})_{6}\right]^{3-}\right)$, an anionic electron acceptor, is repelled to the solution under the effect of electrostatic force, which aids to regenerate $\left[\mathrm{Ru}\left(\mathrm{NH}_{3}\right)_{6}\right]^{3+}$ by oxidizing the produced $\mathrm{Ru}^{\mathrm{II}}$ species $\left.\left(\mathrm{Ru}\left(\mathrm{NH}_{3}\right)_{6}\right]^{2+}\right)$ in the precedent step, thereby amplifying the electrochemical readout. The magnitude of current signal is correlated with the amount of accessible aptamers. All electrochemical measurements were repeated for a minimum of four times for each sensor device or experimental condition.

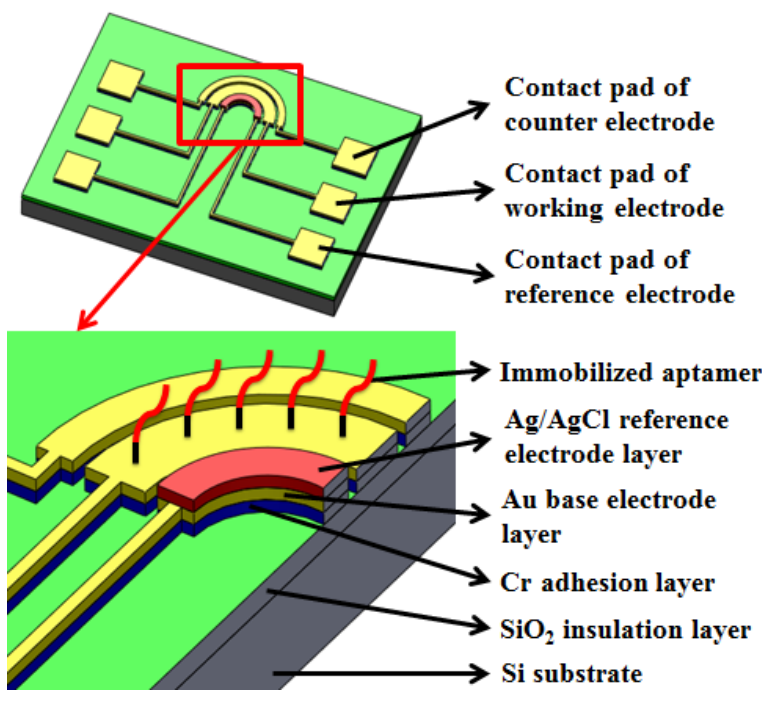

Fig. 1. Schematic drawing to show different layers of proposed MEMS electrochemical aptasensor.

(a)

(b)
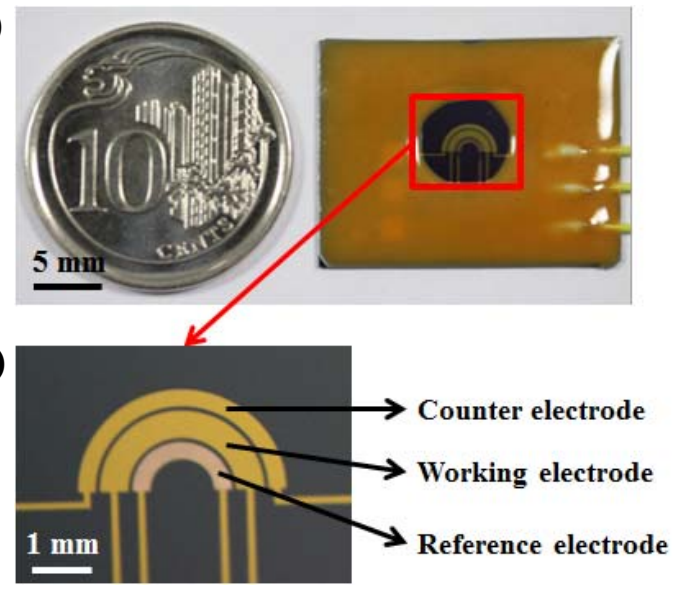

Fig. 2. (a) The MEMS electrochemical aptasensor after fabrication and (b) enlarged view to show on-chip three-electrode configuration.

(a)

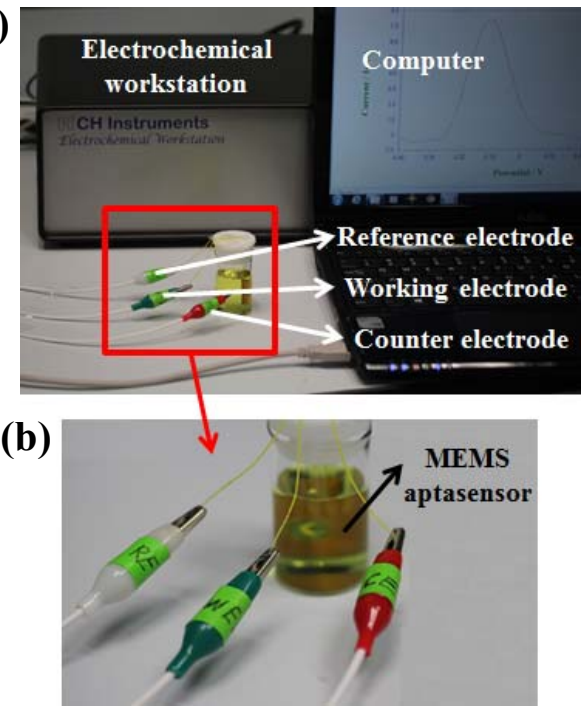

Fig. 3. (a) Photograph of experimental setup for electrochemical investigation and (b) enlarged view to show proposed MEMS electrochemical aptasensor immersed in the testing solution. 


\section{RESULTS AND DISCUSSION}

\section{A. Visual confirmation of aptamer immobilization}

A MEMS aptasensor was prepared by immobilizing an MNV-specific aptamer on the gold working electrode. In order to confirm successful aptamer immobilization, a fluorescentlabeled aptamer was used for immobilization, which allowed visual confirmation of self-assembled aptamer monolayer formed on the electrode surface. As shown in Fig. 4, fluorescent signal derived from the labeled aptamer was observed only on the gold electrode surface, proving that the immobilization procedure employed in this study produced self-assembled aptamer monolayer as expected.
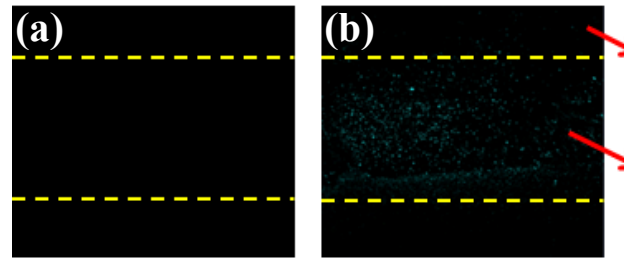

$\mathrm{SiO}_{2}$

substrate surface

Au working

electrode surface

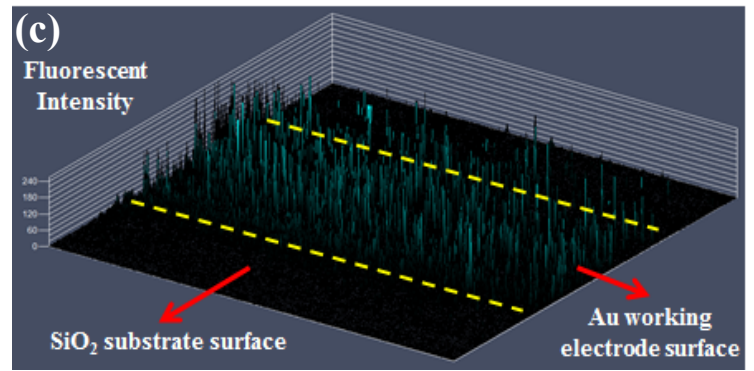

Fig. 4. Images under a laser scanning fluorescent confocal microscope (a) before and (b) after immobilization of fluorescent-labeled aptamer, and (c) intensity of fluorescence ( $z$-axis) derived from the fluorescent-labeled aptamer immobilized on the surface of gold working electrode.

\section{B. Electrochemical evaluation of aptamer immobiliaztion}

The electrochemical characteristics of aptamer immobilization on the working electrode were investigated by $\mathrm{CV}$ examining $\left[\mathrm{Fe}(\mathrm{CN})_{6}\right]^{3-2-}$ redox couple. As shown in Fig. 5, the redox current for the sensor after immobilizing aptamers (red line) showed significant decrease of current magnitude in comparison with the untreated sensor that presented a quasireversible voltammogram with very large redox currents (without aptamer, black line). This result, from the electrochemical point of view, confirmed the successful formation of thiolated aptamer monolayer on the electrode surface, as decrease of oxidation and reduction currents was mainly contributed by the blocking of active area of the working electrode.

Visualization and electrochemical (i.e., CV) evaluation of aptamer immobilization confirmed that MEMS electrochemical aptasensor was successfully fabricated via self-assembly of a thiolated aptamer on the gold working electrode surface following a procedure reported previously [3].

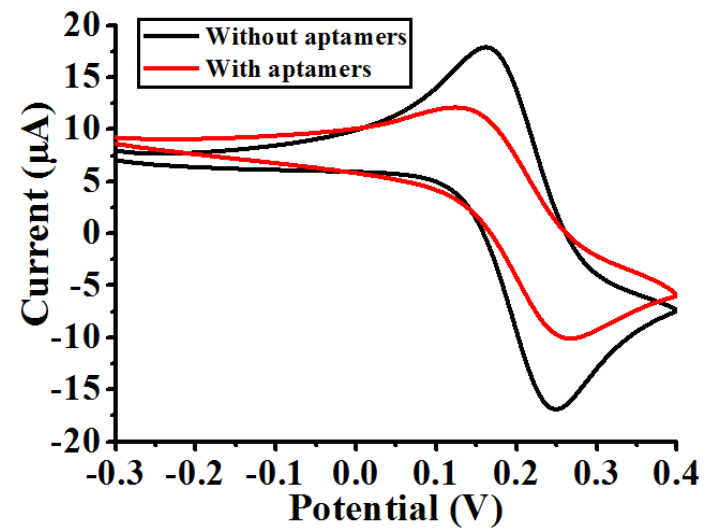

Fig. 5. Comparison of cyclic voltammograms in the solution of $25 \mathrm{mM}$ phosphate buffer ( $\mathrm{pH} 7)$ containing $4 \quad \mathrm{mM} \mathrm{K}_{3}\left[\mathrm{Fe}(\mathrm{CN})_{6}\right]$ and $10 \mu \mathrm{M}$ $\left[\mathrm{Ru}\left(\mathrm{NH}_{3}\right)_{6}\right] \mathrm{Cl}_{3}$ for the MEMS electrochemical aptasensor without (black line) and with (red line) aptamer immobilization.

\section{Electrochemical investigation of MNV binding}

The response of MEMS electrochemical aptasensor upon binding between immobilized aptamers and purified MNV particles was studied with SWV in the solution of $25 \mathrm{mM}$ phosphate buffer ( $\mathrm{pH} \mathrm{7)}$ containing $4 \mathrm{mM} \mathrm{K}_{3}\left[\mathrm{Fe}(\mathrm{CN})_{6}\right]$ and 10 $\mu \mathrm{M}\left[\mathrm{Ru}\left(\mathrm{NH}_{3}\right)_{6}\right] \mathrm{Cl}_{3}$. As shown in Fig. 6, a significant decrease in the magnitude of peak current was observed immediately after MNV was added to the testing solution to obtain a final titer of 50 plaque-forming units per milliliter $(\mathrm{PFU} / \mathrm{ml})$. This result demonstrated that MNV particles were quickly captured by the immobilized aptamer, resulting in reduced amount of accessible aptamers. This experiment also manifested strong affinity of the AG3 aptamer towards MNV particles, as reported in a previous study [3].

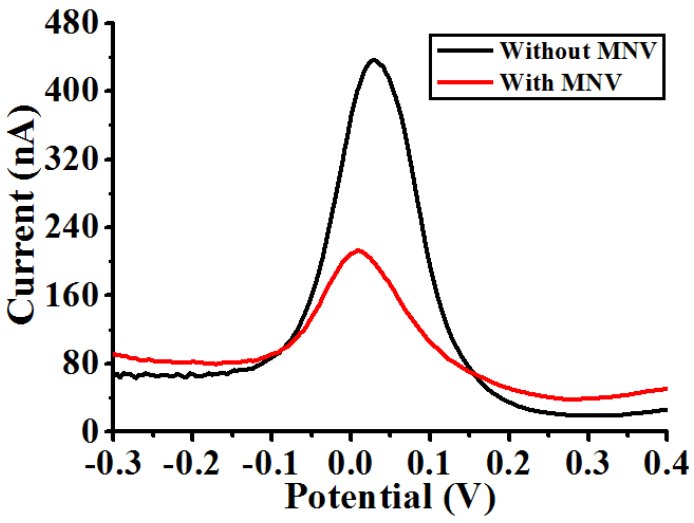

Fig. 6. Comparison of square wave voltammograms in the solution of $25 \mathrm{mM}$ phosphate buffer $\left(\mathrm{pH}\right.$ 7) containing $4 \quad \mathrm{mM} \mathrm{K}_{3}\left[\mathrm{Fe}(\mathrm{CN})_{6}\right]$ and $10 \mu \mathrm{M}$ $\left[\mathrm{Ru}\left(\mathrm{NH}_{3}\right)_{6}\right] \mathrm{Cl}_{3}$ for the MEMS electrochemical aptasensor without (black line) and with (red line) $50 \mathrm{PFU} / \mathrm{ml} \mathrm{MNV}$ addition.

\section{MNV titer-dependent response of the MEMS aptasenor}

In order to evaluate the potential ability of the MEMS electrochemical aptasensor for virus particle quantification, a series of ten-fold dilutions of MNV (0 to $\left.1.0 \times 10^{4} \mathrm{PFU} / \mathrm{ml}\right)$ were prepared and separate SWV measurements using the MEMS aptasensor were performed for each virus titer. As shown in Fig. 7(a), well-defined and legible peaks were obtained, and the magnitude of peak current decreased in a 
MNV titer-dependent manner. More specifically, the magnitude of peak current relative to a baseline, decreased linearly with increasing concentrations of MNV over four orders of magnitude (Fig. 7(b)), with the regression equation of $y=-6.06 x+77.04\left(\mathrm{R}^{2}=0.98\right)$, where $y$ is the magnitude of peak current relative to a baseline (nA) and $x$ is the titer of inoculated $\mathrm{MNV}(\mathrm{PFU} / \mathrm{ml})$ in $\log _{10}$ scale. This result demonstrated high sensitivity and good linearity of the sensor response against different titers of $\mathrm{MNV}$, indicating promising capability of proposed MEMS electrochemical aptasensor for MNV quantification.
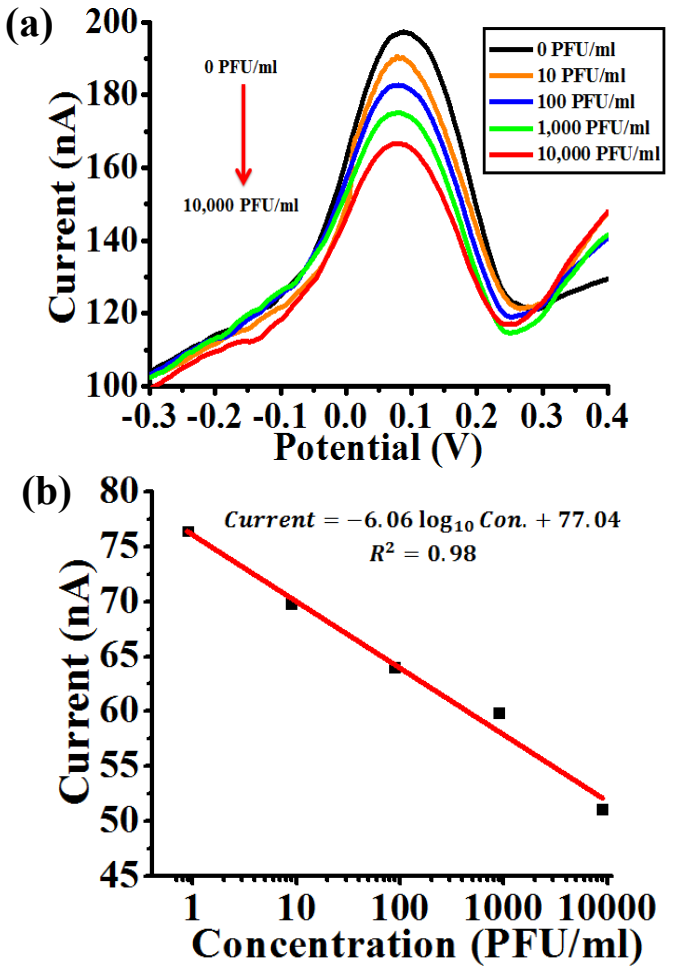

Fig. 7. (a) Square wave voltammograms in the solution of $25 \mathrm{mM}$ phosphate buffer (pH 7) containing $4 \mathrm{mM} \mathrm{K}_{3}\left[\mathrm{Fe}(\mathrm{CN})_{6}\right]$ and $10 \mu \mathrm{M}\left[\mathrm{Ru}\left(\mathrm{NH}_{3}\right)_{6}\right] \mathrm{Cl}_{3}$ with a series of ten-fold dilutions of $\mathrm{MNV}\left(0\right.$ to $\left.1.0 \times 10^{4} \mathrm{PFU} / \mathrm{ml}\right)$ and (b) the corresponding calibration curve of peak current versus MNV concentration.

\section{CONCLUSIONS}

In this work, a miniaturized MEMS electrochemical aptasensor was fabricated, characterized and evaluated for NoV detection and quantification, using MNV as a model virus. The working electrode of the sensor was modified by drop casting an MNV-specific thiolated aptamer, which immobilized the aptamer on the on-chip sensing electrode. Formation of an aptamer monolayer (i.e., successful immobilization of the aptamer on the gold working electrode surface) was confirmed by means of electrochemical measurement as well as visual observation of a fluorescent-labeled aptamer. The MEMS electrochemical aptasensor exhibited rapid and clear response against different titers of MNV, demonstrating its potential for simple, sensitive and rapid NoV detection and quantification. This research forms initial basis for the development of an electrochemical MEMS-aptasensor platform and its application for virus detection. It is anticipated that this MEMS aptasensor is simple and portable enough for on-site NoV detection and automation and such electrochemical MEMS-aptasensor platform can be applicable to detection of other microbial pathogens. Our future efforts will focus on more detailed evaluations of the proposed sensor, in terms of sensitivity, specificity, applicability for environmental samples and further miniaturization of the electrochemical measurement system towards prototyping a portable aptasensor.

\section{ACKNOWLEDGMENT}

This research was supported by the Singapore Ministry of Education and the Singapore National Research Foundation through the Singapore MIT Alliance for Research and Technology's Center for Environmental Sensing and Modeling (CENSAM) interdisciplinary research program. The authors acknowledge the CENSAM Research Initiative program for providing the funding and opportunity to carry out this research.

\section{REFERENCES}

[1] S. Butot, S. Zuber, and L. Baert, "Sample preparation prior to molecular amplification: Complexities and opportunities," Current Opinion in Virology, vol. 4, pp. 66-70, Feb. 2014.

[2] M. Labib, and M. V. Berezovski, "Electrochemical aptasensors for microbial and viral pathogens," Advances in Biochemical EngineeringBiotechnology, vol. 140, pp. 155-181, 2014.

[3] A. Giamberardino, M. Labib, E. M. Hassan, J. A. Tetro, S. Springthorpe, S. A. Sattar, M. V. Berezovski, and M. C. DeRosa, "Ultrasensitive norovirus detection using DNA aptasensor technology," Plos One, vol. 8, no. 11, e79087, Nov. 2013.

[4] C. E. Wobus, S. M. Karst, L. B. Thackray, K. O. Chang, S. V. Sosnovtsev, G. Belliot, A. Krug, J. M. Mackenzie, K. Y. Green, and H. W. Virgin, "Replication of Norovirus in cell culture reveals a tropism for dendritic cells and macrophages," Plos Biology, vol. 2, no. 12, e432, Dec. 2004.

[5] B. I. Escudero-Abarca, S. H. Suh, M. D. Moore, H. P. Dwivedi, and L. A. Jaykus, "Selection, characterization and application of nucleic acid aptamers for the capture and detection of human norovirus strains," Plos One, vol. 9, no. 9, e106805, Sep. 2014.

[6] R. Gasparac, B. J. Taft, M. A. Lapierre-Devlin, A. D. Lazareck, J. M. $\mathrm{Xu}$, and S. O. Kelley, "Ultrasensitive electrocatalytic DNA detection at two- and three-dimensional nanoelectrodes," Journal of the American Chemical Society, vol. 126, no. 39, pp. 12270-12271, Oct. 2004. 